Friedrich Nietzsche / Franz und Ida Overbeck Briefwechsel 


\section{Friedrich Nietzsche / Franz und Ida Overbeck Briefwechsel}

Herausgegeben von Katrin Meyer und Barbara von Reibnitz

J.B. Metzler Stuttgart $\cdot$ Weimar 
Die Deutsche Bibliothek - CIP-Einheitsaufnahme

\section{Nietzsche, Friedrich:}

Briefwechsel / Friedrich Nietzsche, Franz und Ida Overbeck. Hrsg. von Katrin Meyer und Barbara von Reibnitz. - Stuttgart ; Weimar : Metzler, 2000

ISBN 978-3-476-01617-1

ISBN 978-3-476-05240-7 (eBook)

DOI 10.1007/978-3-476-05240-7

Dieses Werk einschließlich aller seiner Teile ist urheberrechtlich geschützt. Jede Verwertung außerhalb der engen Grenzen des Urheberrechtsgesetzes ist ohne Zustimmung des Verlages unzulässig und strafbar. Das gilt insbesondere für Vervielfältigungen, Übersetzungen, Mikroverfilmungen und die Einspeicherung und Verarbeitung in elektronischen Systemen.

(C) 2000 Springer-Verlag GmbH Deutschland

Ursprünglich erschienen bei J. B. Metzlersche Verlagsbuchhandlung und Carl Ernst Poeschel Verlag GmbH in Stuttgart 2000 


\section{Inhalt}

Einleitung VII

Editorische Notiz XIX

Anmerkungen zur Einleitung XXII

Briefverzeichnis XXV

Briefe 1

Anhang

Anmerkungen zum Text 435

Erläuterungen 442

Verzeichnis der in den Erläuterungen benutzten

Abkürzungen 504

Verzeichnis der nicht erhaltenen Briefe 507

Namens- und Werkregister 511 


\section{Einleitung}

\section{Zusammen leben}

»Nächsten Sonntag werde ich wahrscheinlich noch etwas von Basel weggehen, zusammen mit Overbeck ", schrieb Nietzsche im Herbst 1875 an die gemeinsame Freundin Marie Baumgartner im badischen Lörrach. »Es verlangt mich den Pilatus zu sehen und einiges mit Overbeck zusammen auszudenken, von dem niemand nichts weiss und wissen wird. « ${ }^{1}$ Was die beiden Männer sich damals ausgedacht haben, als sie sich auf dem Bürgenstock hoch über dem Vierwaldstättersee einquartierten, erfuhr tatsächlich niemand. Das Datum jedoch markiert eine Wende in den Beziehungen der beiden "Tisch-, Haus- und Gedankenfreunde ", die seit dem Frühjahr 1870 in der »Baumannshöhle « in Basel, unter der bürgerlichen Adresse »Schützengraben 45«, zusammen gewohnt und gelebt hatten. ${ }^{2}$

Overbeck, ein Jahr nach Nietzsche als Professor für Neues Testament und alte Kirchengeschichte von Jena nach Basel berufen und ins gleiche Haus wie Nietzsche gezogen, hatte sich rasch mit dem jungen Professor für Klassische Philologie angefreundet, der eben seine ersten Semester hinter sich gebracht hatte. Anknüpfungspunkte boten nicht nur die sich berührenden Forschungsgebiete, antike Kultur und frühes Christentum, sondern auch die gemeinsame Herkunft aus Sachsen, gemeinsame Bekannte in Leipzig, das Interesse für Richard Wagner und für Schopenhauer - sowie, auch und vor allem, Politik und Zeitkritik. Die Beobachtung und Kritik der deutschen Zustände, zumal nach dem deutsch-französischen Krieg und der Reichsgründung 1871 bildeten, nach Overbecks späterem Zeugnis, den weitaus überwiegenden Gegenstand ihrer »kaum stockenden « täglichen Gespräche. ${ }^{3}$ 
Wand an Wand mit Overbeck hatte Nietzsche 1871 die »Geburt der Tragödie aus dem Geiste der Musik « geschrieben, hier waren ein Jahr darauf die Vorträge »Über die Zukunft unserer Bildungsanstalten « und die »Unzeitgemässen Betrachtungen " entstanden. Overbeck hatte an Nietzsches schriftstellerischen Unternehmungen dieser Jahre nicht nur beobachtend Teil, er trug auch selbst sein Scherflein bei, um den kulturreformerischen Absichten Rechnung zu tragen, die man damals in der "Gesellschaft der Hoffenden « entwikkelte. ${ }^{4}$ Im Frühjahr 1873 verfasste er seine »Streit- und Friedensschrift « »Ueber die Christlichkeit unserer heutigen Theologie«. Seine Argumentation zielte auf die Trennung von Theologie und Kirche, von Religion und Politik, von Glauben und Wissenschaft - im Sinne einer aufgeklärten Kultur und einer Gesellschaft, deren Selbstverständnis keiner religiösen Legitimation mehr bedürfen sollte. Seine Thesen entwickelte er in kritischer Auseinandersetzung mit den beiden Hauptvertretern einer ssäkularen Religion`, Paul de Lagarde und David Friedrich Strauss. Gegen den Letztgenannten ergriff Nietzsche zur gleichen Zeit in seiner ersten Unzeitgemässen Betrachtung die Feder. ${ }^{5}$

In den wenigen Briefen, die Nietzsche und Overbeck in diesen ersten Jahren wechselten, - sie überbrücken lediglich die Abwesenheiten des einen oder des anderen während der Semesterferien - ist all das vorausgesetzt, denn es gehörte zum alltäglichen Miteinanderleben und -sprechen in der Baumannshöhle. Deutlich aber spiegeln die Briefe die Grundstimmung dieser Zeit: in ihnen herrscht die Lust am Sprachspiel und an der polemischen Pointe, sie haben ironischen Schwung und einen bisweilen übermütigen Klang. Die Töne der Angestrengtheit und der An-, ja der Überspannung, wie sie, zumindest für Nietzsches Briefe in den späteren Jahren charakteristisch wurden, sind hier nur selten und verdeckt hörbar.

Dabei darf nicht vergessen werden, dass das ketzerische Schrifttum der Wunsch- Waffen- und Wandnachbarn ${ }^{6}{ }^{6}$ der spärlichen Freizeit abgenötigt war, die den jungen Professoren 
neben ihrem akademischen Amt übrig blieb. Nietzsche musste sich von Semester zu Semester seine Vorlesungen erarbeiten ${ }^{7}$ und auch Overbeck konnte nur auf weniges Material aus seiner Jenenser Privatdozentenzeit zurückgreifen. ${ }^{8}$ Die Arbeitsbelastung und der Ehrgeiz, nicht nur den Forderungen des Amts zu genügen, sondern sich auch als kultur- und gesellschaftskritische Intellektuelle Gehör zu verschaffen, überspannte beider Kräfte innerhalb weniger Jahre. Bereits 1871 - und hier setzt auch der Briefwechsel ein - musste Nietzsche sich wegen andauernder schwerster Kopf- und Magenbeschwerden beurlauben lassen und in Lugano Erholung suchen. Die folgenden Jahre blieben vom Kampf um die bereits zerrüttete Gesundheit geprägt. Overbeck hielt bis zum Sommersemester 1875 durch, das er als Kurgast in Karlsbad verbringen musste.

Damals rief Nietzsche die Schwester zu Hilfe und teilte schliesslich dem einigermassen überraschten Overbeck mit, er werde die Baumannshöhle verlassen und mit Elisabeth einen eigenen Hausstand gründen - dies vor allem, weil seine Gesundheit eines fürsorglichen, den Alltag entlastenden >Regiments bedürfe. Overbeck hatte sich drein zu finden, er wünschte - schweren Herzens - Glück und gutes Gelingen. Im August 1875 zog Nietzsche »um die Ecke « an den Spalentorweg 48. Der herbstliche Aufenthalt der beiden Freunde auf dem Bürgenstock, mit Blick auf den Pilatus und - nicht zu vergessen auf Tribschen, den ehemaligen Wohnsitz Wagners -, war also so etwas wie ein Abschiedsausflug.

Overbeck seinerseits beschloss kurzerhand und kurz darauf, sich zu verloben und ebenfalls einen eigenen Hausstand $\mathrm{zu}$ gründen. Schon seit drei Jahren war er hin und wieder im Hause eines Bekannten in Zürich Ida Rothpletz begegnet. Am 18. Januar 1876 teilte er seine Verlobung mit und schrieb wenige Tage später an Treitschke, er habe sich damit $\gg z u$ einem sehr glücklichen Menschen gemacht «. Am 8. August fand die Hochzeit statt. Wenig ist über Ida Overbeck bekannt, die, wie es scheint, eine ausserordentlich interessante Frau gewesen ist. Elf Jahre jünger als ihr Mann, wurde sie als 
Tochter eines Schweizers und einer Deutschen am 3. Oktober 1848 in der Pfalz geboren und ist dort aufgewachsen. Nach der Scheidung der Eltern lebte sie seit 1868 mit der Mutter und fünf Geschwistern in Zürich. Overbeck mag sich ihr hingezogen gefühlt haben nicht nur, weil sie ein, wie er schrieb, "geist- und gemüthvolles Mädchen « war, klug und hochgebildet, sondern auch, weil sie, wie seine Mutter, eine gebürtige Französin, fliessend französisch sprach. Zudem war sie musikalisch und hatte ein sehr gute Klavierausbildung erhalten - u. a. durch Robert Freund in Zürich. Gemeinsames Musizieren und gemeinsame Lektüre scheint denn auch ein Lebenselement ihrer kinderlos gebliebenen Ehe gewesen zu sein. Nach aussen hielt Ida Overbeck sich als Ehefrau und Gesprächspartnerin ihres Mannes traditionell im »Hintergrund «. ${ }^{9}$ In ihrem Tagebuch vermerkt sie, nicht ohne resignierten Unterton: "Und dazu lebe ich stille, habe fast keine Eindrücke von aussen u. wohne doch den Gedanken vieler Menschen in Büchern bei. «Eben dieses Tagebuch zeigt jedoch auch, dass ihr solche Zurücknahme bei ihrem scharfen und kritischen Intellekt nicht leicht geworden ist. ${ }^{10}$

Overbecks zogen in die Eulerstrasse 53. Mit dem nahe gelegenen Spalentorweg stand man in enger Verbindung. Schliesslich war Ida Overbeck mit Nietzsche länger bekannt als mit ihrem Mann. 1870 hatte sie ihn während einer Sommerkur im Maderanertal kennengelernt. Nietzsche schrieb damals an der »Dionysischen Weltanschauung « - einer Vorarbeit zur "Geburt der Tragödie«. Abends wurde musiziert: Ida und ihre Mutter spielten Nietzsche Brahms und Beethoven vor, er revanchierte sich mit Wagners »Meistersingern «. »Er trug es frei vor ... Er besass keinerlei Virtuosität « erinnerte sich Ida Overbeck sehr viel später: »Er spielte hart und eckig, suchte die Töne in der Erinnerung und dann auf den Tasten «. Gleichwohl - sie war offenkundig beeindruckt von dem jungen Professor. »Ich hatte in Nietzsches Nähe das Gefühl eines Räthsels, eines Geheimnisses. Etwas von mädchenhafter Natürlichkeit begegnete er mit einer gewissen Feierlichkeit, deren Rückschlag bescheidener Humor und Übermut waren. Ich sehe ihn noch die Waldwege gehen, 
rüstig, aber doch den Weg suchend, was seinem Gang ein gewisses ungeschicktes, unfreies Gepräge gab. Ja, er war sicherlich ein Schulmeister, aber man hätte gern Näheres über ihn gewusst. " ${ }^{11}$ Sie verschaffte sich dann sowohl die »Geburt der Tragödie « als auch die »Unzeitgemässen Betrachtungen «, scheint Nietzsche jedoch bis zu ihrer Eheschliessung nicht wiedergesehen und keine Briefe mit ihm gewechselt zu haben.

In der Eulerstrasse knüpften sich die Beziehungen rasch neu und Ida Overbeck wurde in die Gespräche der beiden Freunde sehr weitgehend einbezogen. Ihre »Erinnerungen « geben Aufschluss über gemeinsame Themen und Lektüren, enthalten aber auch manche kritische Bemerkung. Anders, als es der Ton ihrer Briefe vermuten lässt, scheint Ida Overbeck einen scharf pointierenden, bisweilen auch sarkastischen $\mathrm{Hu}-$ mor besessen zu haben und liess sich nicht gern blenden. In ihrem Tagebuch ging sie denn auch, in eher halbherzig klingender Selbstanklage, mit sich ins Gericht wegen der »Bosheit «, die sie »gegen geistreiche Männer« erfülle - denen sie »das Schweigen auch manchmal empfehlen« möchte. »Die Kerle wissen so viel u. verrameln sich dahinter u. hinter Ihrem eignen Ich wie hinter einer Festung. Die Energie mit der sie dies Geschäft betreiben bewundere ich wenn ich auch die feste Überzeugung habe dass sie einiges sehr gut machen $u$. in vieles pfuschen u. es nicht merken. Aber das Pathos u. gelegentlich die Grobheit! « ${ }^{12}$ Eine Freundin, die Ida Overbeck in ihren alten Tagen ${ }^{13}$ zu besuchen pflegte, erinnerte sich zumal an ihre »lebhaften Augen ", die »vor Vergnügen über einen gut sitzenden Hieb, der einem Lebenden oder Toten als 'zu leicht Befundenem ' galt - intensiv funkelten $« .{ }^{14}$ Mit ihrem Mann teilte Ida Overbeck die Vorliebe für den Geist des 18. Jahrhunderts und die Distanz zum Christentum, jedenfalls in seiner kirchlichen Form: seine »eheliche Mit-Heidin « hat Overbeck sie genannt. Wenn Nietzsche von ihr als von einer " A Ausnahme in hundert Stücken « sprach, dürfte er das ernst gemeint haben. ${ }^{15}$

Der nachbarschaftliche Austausch zu dritt, für den Ida Overbecks »Erinnerungen « eine interessante Quelle sind, half 
jedoch nicht gegen die Schatten von Nietzsches Krankheit. Vom Herbst 1876 bis Herbst 1877 musste er für zunächst ein Jahr beurlaubt werden. Im Frühjahr 1879 suchte er bei den Basler Behörden um seine definitive Entlassung aus dem Universitätsdienst nach. In der nun folgenden Zeit wurden abgesehen von seltenen Besuchen oder Begegnungen an drittem Ort - die Briefe das hauptsächliche Medium des Freundschaftsaustauschs.

\section{Diesseits und jenseits des Gotthard}

1879 begann Nietzsche seine Wanderexistenz, immer auf der Suche nach einem Ort, der seinen Augen, seinem Magen, seinem Kreislauf, seinem Kopf gleichermassen zuträglich wäre - im Sommer in den Bergen, im Winter in den Städten des Südens, in Venedig, in Genua, in Nizza, in Turin. Overbeck sorgte von nun an für den existentiellen Rückhalt. Er verwaltete Nietzsches Gelder, d.h. die Pensionszahlungen, die ihm Stadt und Universität Basel in grosszügiger Weise gewährten. Overbeck wurde für Nietzsche »der Postmeister, der Kommissionär, der Bankier, der Arzt, der Vermittler, der Nachrichtenüberbringer, der ewige Tröster, der sanfte Beruhiger « wie Stefan Zweig es in seinem Porträt des Briefwechsels beschrieben hat. ${ }^{16} \mathrm{Z}$ weig hat auch darauf aufmerksam gemacht, wieviel die Lektüre dieses Briefwechsels zur Entheroisierung Nietzsches beitragen könne - weil soviel "Lebenswirklichkeit « in ihn eingegangen sei: eine Wirklichkeit, die geprägt war von Krankheit, Einsamkeit und Selbstüberforderung. Overbeck gegenüber hat Nietzsche sich rückhaltlos geäussert, ihm ohne Unterschied seelische und körperliche Leiden geklagt - ohne doch dabei das Gefühl dafür zu verlieren, was er dem Freund damit zumutete. Immer wieder brach er Ansätze zu Klage oder Überhebung ab - um Vertrautheit und Respekt im Briefwechsel nicht aus der Balance zu bringen. »Verzeih lieber Freund « - mit dieser Bitte enden viele der nach Basel adressierten Briefe und Karten.

Doch nicht nur von Krankheit und Kränkung handelt dieser Briefwechsel, auch von Lektüren ist die Rede, von wissen- 
schaftlichen Projekten und von Werkplänen. Overbeck und Nietzsche haben sich z.B. früh für die russische Literatur interessiert, als diese in den achtziger Jahren ihre erste grosse Rezeptionswelle im deutschsprachigen Raum erlebte. Es werden gemeinsame Themen skizziert, wie das Projekt einer Untersuchung über die 'Freundschaft im Altertum ${ }^{17}$ oder das einer 'Kritik des deutschen Obskurantismus - von dem Overbeck allerdings abgeraten zu haben scheint. ${ }^{18}$ Nietzsche kommt immer wieder auf die anhaltende Irritation zu sprechen, die für ihn in der gescheiterten Beziehung zu Richard Wagner lag. Von alten Bekannten, zumal aus der Basler Zeit, und von neuen Begegnungen, etwa mit Heinrich von Stein oder mit Josef Paneth aus Wien ist die Rede, sowie nicht zuletzt von den politischen Verhältnissen und Zeitströmungen. Dass die Freunde die Ende der siebziger Jahre einsetzende antisemitische Agitation aufmerksam verfolgten, wird kaum überraschen, denn beide waren durch ihren gemeinsamen Verleger Schmeitzner persönlich involviert und Nietzsche hatte sich obendrein von seinem antisemitisch engagierten Schwager abzugrenzen.

Leider ist ein grosser Teil der Briefe Overbecks nicht erhalten, sodass seine Stimme und seine Interessen sich in der vorliegenden Korrespondenz nicht gleichgewichtig aussprechen. Doch auch die überlieferten Briefe zeigen, dass seine Meinungen über Nietzsches Leben und Werk pointiert und differenziert waren, reicher als es das Bild des snie erbitterten, nie ungeduldigen, nie gereizten ‘ Freundes (Zweig), der gegenüber Nietzsche oft aus Zartsinn und Respekt schweigt, vermuten liesse. Overbeck ist nicht nur Nietzsches »zweite und bessere Vernunft «, wie dieser sagt, ${ }^{19}$ und er verkörpert nicht nur, im Hinblick auf Nietzsches geistige Isoliertheit, was Walter Benjamin als den »Repräsentanten einer einsichtsvolleren Nachwelt ${ }^{20}$ bezeichnet hat, sondern bleibt dabei ein eigenständiger Denker, der weder in der Rolle des Mitstreiters, noch der des Schülers oder gar Meisters aufgeht. Deutlich spricht sich das aus in seinem Antwortbrief auf Nietzsches "Genealogie der Moral «: Seinem eigenen Selbstverständnis als Gelehrter will vor allem die »Stärke des Affekts« 
in einer »historischen Betrachtungsweise « »nicht eingehen $\ll^{21}$.

Ida Overbeck ist in den Briefwechsel als mitangesprochene und mitsprechende Dritte, als eigenständige Vertraute Nietzsches einbezogen. Wenn es einmal Overbeck gegenüber heisst: "Dieser Brief ist für dich allein ${ }^{22}$, so bestätigt diese Ausnahme gerade die Regel. Nicht zufällig wird diese doppelte Adressatenschaft in Nietzsches letzter "Botschaft " explizit: "Dem Freunde Overbeck und Frau « ist der Brief vom 4. Januar 1889 überschrieben. ${ }^{23}$ Als eigenständige Gesprächspartnerin Nietzsches zeigt sie sich in ihren Übersetzungen, die sie teils auf seine Anregung hin angefertigt hat, wie die Auswahl aus den "Causeries du Lundi« von Sainte-Beuve, teils in konvergierendem Interesse sich vornahm, wie diejenige der Essays von Emerson. Nietzsche scheint ihrem psychologischen Feingefühl und ihrer Aufgeschlossenheit so sehr vertraut zu haben, dass er sie zur Vertrauten im Hinblick auf seine Beziehung zu Lou von Salomé machte und sie bat, mit der jungen Frau über (und für?) ihn zu sprechen. Wie sie diese Aufgabe verstand und wahrnahm, darüber hat Ida Overbeck sich in ihren "Erinnerungen ", aber auch in ihrem Tagebuch geäussert. Es scheint, als habe sie das intellektuelle und persönliche Freiheitsbedürfnis Lou von Salomés geschätzt und unterstützt. So ist es kaum verwunderlich, dass sie wiederum mit Elisabeth Nietzsche in ein gespanntes Verhältnis kommen musste. Diese intrigierte von da an heftig gegen Ida Overbeck und da Nietzsche in Beziehung auf seine Familie alles andere als konsequent war, scheint sein Verhältnis zu Ida Overbeck die 'Lou-Affäre nicht unbeschadet überstanden zu haben. ${ }^{24}$ Es wird kaum ein Zufall sein, dass Nietzsche nach 1883 nicht mehr direkt an sie geschrieben hat und dass er seitdem auch nicht mehr zu Besuch nach Basel gekommen ist.

Diese Spannungen hat Overbeck klug und behutsam auszugleichen versucht. Dennoch zeigen seine Briefe der späten achtziger Jahre eine zunehmende Reserve, gleichsam ein >Abblenden s seiner Wahrnehmungssensibilität angesichts der sich steigernden Intensität und Radikalität von Nietzsches Selbst- 
beschreibungen und Werkproduktivität. Dieses durch eigene Krankheit mitbedingte Sich-Abblenden gegenüber dem Freund mag dazu beigetragen haben, dass ihn Nietzsches Zusammenbruch an der Wende des Jahres 1889 mit der doppelten Wucht verdrängter Beunruhigung traf.

Am 7. Januar fuhr Overbeck nach Turin und begleitete den kranken Freund zurück nach Basel. Am Morgen des 9. Januars wurde Nietzsche in die dortige psychiatrische Universitätsklinik eingeliefert. Damit endete der unmittelbare Austausch der Freunde in Brief und Gespräch.

Bis zu seinem Tod am 26. Juni 1905 hörte Overbeck jedoch nicht auf, mit Nietzsche sumzugehen ‘. Aus dem retrospektiven Nachdenken über ihre Freundschaft entstanden seine »Aufzeichnungen über Nietzsche «. Sie bildeten, nach seinem eigenen Verständnis, die monologische Fortsetzung des Gesprächs in den Briefen - und richteten sich zugleich gegen den öffentlichen "Betrieb « mit dem und um den Freund. Nicht zuletzt aufgrund seiner konsequenten Abkehr und Fernhaltung von diesem öffentlichen Betrieb, glaubte Overbeck sich zu Nietzsches » wirklichen Freunden « rechnen zu können ${ }^{25}$ - wobei er anfügte, dass es für Nietzsche die »wahren Freunde " sowenig habe geben können wie die "wahre Welt « neben der »wirklichen«. Diese »wirkliche« Freundschaft oder die »Wirklichkeit einer Freundschaft « - ist im vorliegenden Briefwechsel über einen Zeitraum von mehr als siebzehn Jahren dokumentiert.

\section{Nachleben und Editionsgeschichte}

Die Erstveröffentlichung des Nietzsche-Overbeck-Briefwechsels erfolgte spät und ist Teil der problematischen Editionsgeschichte von Nietzsches Werken und Briefen unter der Leitung von Nietzsches Schwester. Overbeck hatte die editorischen Aktivitäten des von ihr 1894 gegründeten NietzscheArchivs ${ }^{26}$ mit wachsendem Unbehagen an der sbetriebsmässigen Geschäftigkeit` und dem 'Diktat der Publizität‘, der sie unterworfen waren, verfolgt. ${ }^{27}$ Dem "Geschäft mit Nietzsche« wollte er seine Briefe nicht nur zu seinen Lebzeiten, 
sondern auch darüber hinaus entzogen wissen. Als sich die Spannungen und Gegensätze verschärften, stellte Overbeck sein eigenes »Nietzsche-Archiv« zusammen. Dessen Kernstück waren die Briefe Nietzsches, ergänzt durch die "Nietzsche-Aufzeichnungen " und ausgewählte weitere Dokumente. ${ }^{28}$ Overbecks Archiv wurde später die Basis des sogenannten "Basler Nietzsche-Archivs «. ${ }^{29}$ Mit der postumen Veröffentlichung seiner Nietzsche-Briefe betraute Overbeck den Freund und Schriftsteller Carl Albrecht Bernoulli, den er als seinen wissenschaftlichen Nachlassverwalter eingesetzt hatte. Nach langjährigem Streit um die Urheberrechte an den Briefen Nietzsches, der Bernoullis Vermögen ruinierte, ${ }^{30}$ wurde 1916 auf der Grundlage eines gerichtlichen Vergleichs die Veröffentlichung des Briefwechsels unter der doppelten Herausgeberschaft zunächst von Wilhelm Nestle, dann von Richard Oehler (Weimar) und Carl Albrecht Bernoulli (Basel) möglich. ${ }^{31}$ In dieser Ausgabe mussten auf Wunsch Elisabeth Förster-Nietzsches und mit Rücksicht auf noch lebende Zeitgenossen verschiedene Stellen ausgelassen werden. Erich F. Podach, der diese gekürzten Stellen 1963 erstmals publiziert hat, ${ }^{32}$ hatte bereits 1935, unmittelbar nach dem Tod Elisabeth Förster-Nietzsches und kurz vor Erscheinen der ersten kritischen Ausgabe ${ }^{33}$, eine vollständige Ausgabe des OverbeckNietzsche-Briefwechsels (einschliesslich der Briefe Ida Overbecks) geplant, um damit gegen die Editionstätigkeit des Weimarer Archivs anzutreten. ${ }^{34}$ Das Unternehmen scheiterte jedoch, da ihm die Universitätsbibliothek Basel als testamentarisch bestimmte Eigentümerin und die Basler Overbeck-Stiftung - vor Podachs allzu streitbarem Ton durch Karl Jaspers gewarnt - nach anfänglicher Einwilligung die Unterstützung entzogen. ${ }^{35}$ Es dauerte bis 1975 , bis sowohl Nietzsches wie auch Franz und Ida Overbecks Briefe in der kritischen Ausgabe von Giorgio Colli und Mazzino Montinari ${ }^{36}$ (KGB) vollständig publiziert zugänglich wurden.

\section{$\mathrm{Zu}$ dieser Ausgabe}

Die vorliegende Ausgabe ist als Leseausgabe konzipiert. Sie will die Korrespondenz zwischen Nietzsche und Franz und 
Ida Overbeck als geschlossenen Briefwechsel zugänglich machen. Während die chronologische Sammlung aller Briefe von und an Nietzsche einen Blick auf Varianz und Redundanz von Nietzsches Selbstbeschreibungen ermöglicht, soll hier mit dem Fokus auf einen einzigen Briefwechsel das verdichtete Gespräch, das sich aus der Korrespondenz zweier oder dreier Personen bildet, im Zentrum stehen. Aus diesem Grund wurde darauf verzichtet, Briefentwürfe Nietzsches aufzunehmen, die den Empfängern unbekannt geblieben sind und sich in den wechselseitigen Austausch der Briefpartner nicht integrieren. ${ }^{37}$

Die Veröffentlichung dieses Briefwechsels erfolgt bewusst parallel zur Veröffentlichung von Overbecks Nietzsche-Aufzeichnungen in Band 7/2 der Overbeck-Werkausgabe. Damit wird den ursprünglichen Absichten Overbecks Rechnung getragen, der diese Aufzeichnungen als Begleittexte zu seinen Nietzsche-Briefen verstanden wissen wollte.

Die Briefe wurden möglichst knapp erläutert. Die Erläuterungen orientieren sich an einer chronologischen Lektüre. Hinweise auf Personen, Ereignisse und Werke werden in der Regel nur an der ersten Stelle ihrer Erwähnung gegeben; die Erläuterungen werden erschlossen und ergänzt durch das Namens- und Werkregister, das auch die biographischen Grunddaten verzeichnet.

Auf eine detaillierte Manuskriptbeschreibung und eine ausführliche wissenschaftliche Kommentierung der Briefe wurde verzichtet, da diese in den Nachberichts-Bänden der Kritischen Gesamtausgabe (KGB) bereits vorliegt oder in den nächsten Jahren zu erwarten ist. ${ }^{38}$

Durch kurze Einschaltungen zwischen den Briefen wird der biographische Hintergrund skizziert, vor dem sich die Korrespondenz entwickelt, sofern er nicht aus dieser selbst hervorgeht. Diese Zwischentexte konzentrieren sich vornehmlich auf Nietzsche, dessen Existenz - anders als der an äusserlichen Ereignissen ruhige Lebensverlauf des Ehepaares Overbeck durch den ständigen Wechsel des Aufenthaltsorts bestimmt und auf die Produktion seiner Werke ausgerichtet war.

Die vorliegende Sammlung umfasst 237 Briefe von Nietz- 
sche, 94 Briefe von Franz Overbeck und 3 Briefe von Ida Overbeck. Eine empfindliche Lücke bilden die über einen Zeitraum von anderthalb Jahren, von Mitte 1880 bis Ende 1881 vollständig, sowie für die Jahre 1882 und 1883 bis auf wenige Ausnahmen verlorenen Briefe Franz und Ida Overbecks an Nietzsche. Das Verzeichnis der verlorenen Briefe im Anhang gibt über Zahl und jeweiliges Datum aller verlorenen, bzw. als verloren zu vermutenden Briefe Aufschluss.

Wir möchten an dieser Stelle der Universitätsbibliothek Basel, an erster Stelle dem Leiter der Handschriftenabteilung, Herrn Prof. Dr. Martin Steinmann, danken, der uns für unsere Arbeit in grosszügigster Weise die Originale der Briefe zur Verfügung gestellt hat. Seine Mitarbeiter und Mitarbeiterinnen haben uns entgegenkommend unterstützt, das gilt nicht zuletzt für Herrn Courvoisier von der Basler Porträtsammlung sowie Frau Schwizer und Herrn Bürgin in der ReproAbteilung der Universitätsbibliothek Basel. Auch den Mitarbeitern des Goethe- und Schiller-Archivs (Weimar), Herrn Ritschel und Frau Ellermann, sowie Herrn von Bülow vom Marbacher Literatur-Archiv sind wir für unkomplizierte und rasche Hilfe sehr zu Dank verpflichtet.

Den Kolleginnen von der Basler Nietzsche-(Brief-)Edition, Franziska Tränkle und Andrea Bollinger, möchten wir ebenso wie Renate Müller-Buck in Tübingen für jederzeit bereitwillig erteilte Auskünfte danken. Marianne Stauffacher, Martin Loppacher und Salome Meyer haben uns beim Lesen der Fahnen, Matteo Serpi beim Erstellen des Registers unterstützt.

Oliver Schütze vom Metzler-Verlag hat das gesamte Projekt sehr engagiert begleitet und Thomas Schröck hat zuverlässig und sorgfältig für die Herstellung gesorgt.

Ohne die stete Ermunterung und sachliche Unterstützung von Niklaus Peter wären wir mit dieser Arbeit jedoch kaum termingerecht zu Ende gekommen.

Katrin Meyer und Barbara von Reibnitz Basel, im September 1999 


\section{Editorische Notiz}

Der Text dieser Ausgabe wurde nach den Handschriften erstellt, unter Beizug der Erstausgabe von Oehler und Bernoulli sowie der Kritischen Ausgabe von Colli und Montinari (KGB). Die Briefe Nietzsches an Franz und Ida Overbeck, ebenso wie die Briefe Overbecks an Nietzsche befinden sich bis auf wenige Ausnahmen im Nachlass Overbeck in der Handschriftenabteilung der Universitätsbibliothek Basel. Alle anderen Fundorte sind in den Anmerkungen zur Kopfzeile der Briefe vermerkt.

Der Text entspricht dem Laut- und Zeichenstand der Handschrift. Wiedergegeben ist in der Regel die letzte Korrekturstufe. Sämtliche Herausgebereingriffe sind in den »Anmerkungen zum Text" verzeichnet.

Von den Auszeichnungen in der Handschrift wurden nur einfache und doppelte Unterstreichungen wiedergegeben; auf die Reproduktion lateinischer Schrift in deutsch geschriebenen Briefen, die vor allem der Verdeutlichung und allenfalls vermittelt der Betonung dient, wurde hingegen verzichtet. Nietzsches Briefe sind anfangs lateinisch oder deutsch geschrieben, von Frühjahr 1879 an aber in der Regel deutsch; solange die Schreibweise wechselt, ist dies jeweils in den Anmerkungen zum Briefkopf verzeichnet. Franz und Ida Overbeck schrieben beide in lateinischer Schrift.

\section{Zur Textkonstitution im einzelnen:}

Ein Problem für die Textkonstitution der Nietzsche-Briefe stellen vor allem die häufigen Binnen- und Endverschleifungen dar. In allen Fällen, wo der Editor nur dezisionistisch verfahren kann, haben wir uns an den Lesungen der KGB orientiert. Auch in der Wiedergabe von $» C$ « und $» \mathrm{~K}$ «, für die 
Nietzsche häufig das gleiche Zeichen verwendet, sind wir den Entscheidungen der KGB gefolgt. Nicht immer eindeutig zu unterscheiden sind Nietzsches Schreibungen für »und « oder »u.«; während im Text der KGB generell aufgelöst wurde, wurde hier nach Lesung unterschieden; ebenfalls nicht eindeutig ist Nietzsches abgekürzte Schreibung für francs: es wurde nach Lesung zwischen frs., frc. und frcs. unterschieden.

Die Namensunterschrift Overbecks wurde, wo sie nicht eindeutig abgekürzt, sondern als Kürzel ausgezogen ist, ausgeschrieben.

Text, der auf die Briefränder geschrieben ist, steht grundsätzlich am Briefende; bei zu vermutendem Textbezug wurde er durch ein in Ergänzungsklammern gesetztes Anmerkungszeichen $\langle *\rangle$ zugeordnet.

Die Briefdatierungen wurden nach dem Original wiedergegeben; stehen sie am Briefende, dann ist das Datum zu Beginn als Ergänzung gesetzt. Bei den zu vermutenden Datierungen haben wir uns an den Vorschlägen der KGB orientiert.

Nicht wiedergegeben wurden Overbecks verschiedene $\mathrm{Nu}$ merierungen der Nietzschebriefe: auf den Briefen findet sich eine durchlaufende Numerierung von 1-226 sowie für die Jahre von 1886 an eine (wohl frühere) jahresweise Zählung.

Nicht wiedergegeben sind Textzusätze von erkennbar fremder Hand.

Notate auf Overbeck-Briefen, die vermutlich von der Hand Nietzsches stammen, sind in den Anmerkungen angegeben.

\section{Zur Textgestalt:}

Briefanrede und -schluss zeigen in der Handschrift zahlreiche Variationen, die sich teils einer Gestaltungsabsicht des Schreibers/der Schreiberin teils aber auch kontingenten Bedingungen wie dem zur Verfügung stehenden Raum verdanken. Zumal die Postkarten verzichten aus Platzgründen meist auf eine gesonderte Anrede- und Schlusszeile. Wir haben bei Briefen und Postkarten die Varianten der Anredezeile durch 
einheitliche Einrückung und nachfolgende Leerzeile wiedergegeben; wo eine solche Anredezeile ganz fehlt, haben wir den Text mit Einzug begonnen. Die Briefschlüsse, die in der Handschrift meist in Relation zum vorstehenden Textfall gestaltet sind, konnten typographisch nicht analog wiedergegeben werden. Es wurde jedoch, wie auch bei den Adressund Datumszeilen des Briefkopfs, der Zeilenfall des Manuskripts beibehalten. In den Overbeck-Briefen fehlt in der Regel die eigentliche Schlusszeile, die Namensunterschrift bildet meist auch den syntaktischen Abschluss des letzten Satzes und steht in der Regel schräg versetzt unter dem letzten Wort. Sie wurde im Drucktext rechtsbündig vereinheitlicht.

Alle Absätze der Handschrift wurden durch Einzüge wiedergegeben; Postscripta und Randtexte hingegen stehen ohne Einzug am Briefende.

Barbara von Reibnitz

Im Text verwendete Zeichen:

$<*>$ : vermuteter Textbezug

[---]: Lücke in der Handschrift 


\section{Anmerkungen zur Einleitung}

1 Nietzsche an Marie Baumgartner, 20. September 1875, KSB 5, S. 111.

2 Vgl. C.A. Bernoulli, Franz Overbeck und Friedrich Nietzsche. Eine Freundschaft, 2 Bde., Jena 1908; B. v. Reibnitz, "Ich verdanke Dir soviel, lieber Freund ...« - Nietzsches Freundschaft mit Franz Overbeck, in: D.M. Hoffmann (Hrsg.), Nietzsche und die Schweiz (Ausstellungskatalog), Zürich 1994, S. 46-54.

3 Vgl. das knappe Porträt ihrer Freundschaft, das Overbeck der 2. Auflage seiner »Christlichkeit « vorangestellt hat (OWN 1, S. 268272).

4 So nannte sich der Freundeskreis, in den neben Overbeck auch Romundt, Rohde und Gersdorff einbezogen waren; zu diesen und weiteren Freunden vgl. H.P. Eberlein, »Flamme bin ich sicherlich «. Friedrich Nietzsche, Franz Overbeck und ihre Freunde, Köln 1999 (im Druck).

5 Vgl. K. Pestalozzi, Overbecks »Schriftchen « »Über die Christlichkeit unserer heutigen Theologie « und Nietzsches "Erste unzeitgemässe Betrachtung: David Friedrich Strauss. Der Bekenner und der Schriftsteller ", in: R. Brändle, E.W. Stegemann (Hrsg.), Franz Overbecks unerledigte Anfragen an das Christentum, München 1988, S. $91-$ 107.

6 Vgl. unten Brief Nr. 7, S. 10.

7 Ein vollständiges Verzeichnis findet sich bei C.P. Janz, Nietzsches akademische Lehrtätigkeit in Basel, in: Nietzsche-Studien 3, 1979, S. 192-203.

$8 \mathrm{Vgl}$. das vollständiges Verzeichnis der Overbeckschen Vorlesungen in Overbeckiana II, S. 181-188.

9 So ist auch ihre Sainte-Beuve-Übersetzung 1880 auf ihren ausdrücklichen Wunsch anonym erschienen, vgl. Brief Nr. 97.

$10 \mathrm{NlO}$, A 265, p. 2; Auszüge des Tagebuches sind abgedruckt in Overbeckiana II, S. 121-124.

11 Ida Overbeck, Erinnerungen an Friedrich Nietzsche, zuerst (gekürzt) in: März, Jg. 1, 1907, Bd. 3, S. 223-235, vollständig in: C.A. Bernoulli: Overbeck-Nietzsche. Eine Freundschaft, Jena 1908, Bd. 1, S. 234-51, S. 336-346; hier: S. 234.

12 NlO, A 265, vgl. Overbeckiana II, S. 121.

13 Ida Overbeck überlebte ihren Mann um fast 20 Jahre und starb am 16. November 1933 in Basel.

14 H. V. v. T., Frau Ida Overbeck zu Hause. Undatierter Zeitungsaus- 
schnitt, inliegend in Ida Overbecks Tagebuch, Nachlass Overbeck, A 265.

15 Vgl. unten Brief Nr. 76, S. 92.

16 Stefan Zweig: Nietzsche und der Freund, in: Insel-Almanach auf das Jahr 1919, Leipzig o.J.

17 Vgl. unten die Briefe Nr. 68 und 69.

18 Vgl. unten Briefe Nr. 210 u. 211.

19 Vgl. unten Brief Nr. 88, S. 106.

20 Walter Benjamin: Deutsche Menschen, in: ders.: Gesammelte Schriften Bd. IV/1, Frankfurt a.M. 1972, S. 228.

21 Vgl. unten Brief Nr. 306, S. 392.

22 Vgl. unten Brief Nr. 202, S. 231.

23 Vgl. unten Brief Nr. 334, S. 432.

24 Verdeckt und verklausuliert klingt das an in den Briefen, die Nietzsche kurz vor dem 14. August 1883 an Franz und Ida Overbeck geschrieben hat.

25 Franz Overbeck: Werke und Nachlaß (OWN), Bd. 7/2: Autobiographisches "Meine Freunde Treitschke, Nietzsche und Rohde", hrsg. von Barbara v. Reibnitz und Marianne Stauffacher-Schaub, Stuttgart, Weimar 1999, S. 59.

26 Vgl. dazu David Marc Hoffmann: Zur Geschichte des NietzscheArchivs, Berlin, New York 1991, S. 18-59.

27 Vgl. die Artikel »Nietzsche-Archiv«, "Nietzsche-Schwester« u.s.w. in OWN $7 / 2$.

$28 \mathrm{Vgl}$. hierzu B. v. Reibnitz, Einleitung zu OWN 7/2, S. XXXIff.

29 D.M. Hoffmann, Das »Basler Nietzsche-Archiv «. Katalog der Ausstellung, Universitätsbibliothek Basel 1993.

30 Gemäss Eva Bernoulli: Erinnerungen an meinen Vater Carl Albrecht Bernoulli 1868-1937, Basel 1987, S. 64 und 67.

31 Friedrich Nietzsches Briefwechsel mit Franz Overbeck, hrsg. v. R. Oehler u. C.A. Bernoulli, Leipzig (Insel) 1916. Vgl. zu den Details die Korrespondenz im Nachlass C.A. Bernoulli (UB Basel, Konvolut G XII) sowie Ulf Diederichs: Kampagne um Nietzsche. Zur Entstehungs- und Wirkungsgeschichte von C. A. Bernoulli, »Franz Overbeck und Friedrich Nietzsche. Eine Freundschaft « (1908), in: Börsenblatt für den deutschen Buchhandel, Nr. 74, Frankfurt und Leipzig, 1994, S. B 99-101 und S. B 112, Anm. 35.

32 Erich F. Podach: Ein Blick in Notizbücher Nietzsches. Ewige Wiederkunft - Wille zur Macht - Ariadne. Eine schaffensanalytische Studie. Heidelberg, 1963, S. 184-190.

33 Friedrich Nietzsche, Briefe. Historisch-Kritische Gesamtausgabe, hrsg. v. W. Hoppe u. K. Schlechta, München 1938-43 (nur 4 Bände erschienen).

34 Vgl. seinen Briefwechsel mit C. A. Bernoulli, Nachlass Bernoulli, UB Basel, Konvolut G VI. 
35 Vgl. D.M. Hoffmann: Das »Basler Nietzsche-Archiv«. Katalog der Ausstellung, Basel 1993, S. 73.

36 Friedrich Nietzsche, Briefwechsel. Kritische Gesamtausgabe, begründet von G. Colli u. M. Montinari, fortgeführt von N. Miller u. A. Pieper, Berlin/New York $1975 \mathrm{ff}$.

37 Diese Entwürfe wurden jedoch für die Erläuterungen herangezogen; die bis dato bekannten Entwürfe sind in der KGB vollständig zugänglich.

38 Bisher sind erschienen die Nachberichte KGB I.4 (1849-1869; bearbeitet von F. Gerratana u. R. Müller-Buck), Berlin/New York 1993 sowie II/7.1 (1869 - Mai 1872; bearbeitet von R. Müller-Buck) Berlin/ New York 1998 und II/7.2 (Mai 1872-1874) bearbeitet von A. Bollinger, K. Meyer, R. Stockmar, H. Thüring, F. Trenkle) Berlin, New York 2000 (im Druck). 


\section{Briefverzeichnis}

1. Nietzsche an Overbeck, nach dem 22. März $1871 \quad 1$

2. Overbeck an Nietzsche, 17. April 18714

3. Nietzsche an Overbeck, 2. Aprilhälfte 18726

4. Overbeck an Nietzsche, 20. April $1872 \quad 6$

5. Overbeck an Nietzsche, 22. April 18727

6. Overbeck an Nietzsche, 22. Dezember $1873 \quad 8$

7. Nietzsche an Overbeck, 31. Dezember 187310

8. Nietzsche an Overbeck, 20. Juli 187411

9. Overbeck an Nietzsche, 26. Juli 187412

10. Nietzsche an Overbeck, 30. Juli 187414

11. Nietzsche an Overbeck, 7./8. August 187416

12. Overbeck an Nietzsche, 10. August $1874 \quad 17$

13. Nietzsche an Overbeck, 2. Oktober 1874 (an Overbeck und H. Romundt) 19

14. Overbeck an Nietzsche, 3. Oktober $1874 \quad 21$

15. Overbeck an Nietzsche, 12. Mai 187522

16. Nietzsche an Overbeck, 21. Mai 187524

17. Overbeck an Nietzsche, 26. Mai $1875 \quad 25$

18. Nietzsche an Overbeck, 30. Mai 187529

19. Overbeck an Nietzsche, 15. Juni 187531

20. Overbeck an Nietzsche, 29. Juni 187533

21. Nietzsche an Overbeck, 14. Juli 187534

22. Overbeck an Nietzsche, 27. Juli 187536

23. Overbeck an Nietzsche, 4. August 187538

24. Nietzsche an Overbeck, 11. August 187540

25. Overbeck an Nietzsche, 13. März 187642

26. Overbeck an Nietzsche, 4. April 187644

27. Nietzsche an Overbeck, 5. April $1876 \quad 46$

28. Overbeck an Nietzsche, 2. August $1876 \quad 47$

29. Overbeck an Nietzsche, 8. September 187648

30. Nietzsche an Overbeck, 11. November 187650

31. Nietzsche an Overbeck, 6. Dezember 187650

32. Overbeck an Nietzsche, 18. Dezember 187651

33. Nietzsche an Overbeck, 26. März $1877 \quad 53$

34. Nietzsche an Overbeck, 7. Mai $1877 \quad 54$

35. Overbeck an Nietzsche, 13. Mai $1877 \quad 55$ 
36. Nietzsche an Overbeck, 1. Juni $1877 \quad 57$

37. Overbeck an Nietzsche, 3. Juni $1877 \quad 57$

38. Nietzsche an Overbeck, 6. Juni $1877 \quad 59$

39. Overbeck an Nietzsche, 8. Juni 187759

40. Nietzsche an Overbeck, 17. Juni 187761

41. Overbeck an Nietzsche, 20. Juni 187761

42. Overbeck an Nietzsche, 29. Juni 187762

43. Nietzsche an Overbeck, 2. Juli 187763

44. Nietzsche an Overbeck, 18. Juli 187763

45. Overbeck an Nietzsche, 30. Juli 187764

46. Nietzsche an Overbeck, 28. August $1877 \quad 65$

47. Overbeck an Nietzsche, 1. September $1877 \quad 67$

48. Nietzsche an Overbeck, 11. September 187768

49. Overbeck an Nietzsche, 23. September $1877 \quad 69$

50. Nietzsche an Overbeck, 25. September $1877 \quad 71$

51. Overbeck an Nietzsche, 9. März 187871

52. Nietzsche an Overbeck, 3. April 187872

53. Nietzsche an Overbeck, 25. August 187873

54. Overbeck an Nietzsche, 29. August 187874

55. Nietzsche an Overbeck, 3. September 187875

56. Nietzsche an Overbeck, 17. September 187876

57. Overbeck an Nietzsche, 19. September $1878 \quad 76$

58. Overbeck an Nietzsche, 13. Oktober $1878 \quad 77$

59. Overbeck an Nietzsche, 31. Dezember 187878

60. Nietzsche an Overbeck, Mitte Januar 187979

61. Nietzsche an Overbeck, 17. März 187979

62. Nietzsche an Overbeck, 23. März 187980

63. Nietzsche an Overbeck, 26. März 187980

64. Overbeck an Nietzsche, 27. März $1879 \quad 81$

65. Nietzsche an Overbeck, 30. März 187982

66. Overbeck an Nietzsche, 1. April 187983

67. Nietzsche an Overbeck, 3. April 187984

68. Overbeck an Nietzsche, 7. April 187984

69. Nietzsche an Overbeck, 11. April 187985

70. Nietzsche an Overbeck, 12. April 187986

71. Nietzsche an Overbeck, 18. April 187986

72. Nietzsche an Overbeck, 8. Juni 187987

73. Nietzsche an Overbeck, 15. Juni 187988

74. Overbeck an Nietzsche, 17. Juni $1879 \quad 89$

75. Overbeck an Nietzsche, 19. Juni 187990

76. Nietzsche an Overbeck, 23. Juni 187991

77. Overbeck an Nietzsche, 25. Juni 187992

78. Nietzsche an Franz und Ida Overbeck, 11. Juli 187994

79. Overbeck an Nietzsche, 19. Juli 187994

80. Nietzsche an Overbeck, 31. Juli 187996 
81. Overbeck an Nietzsche, 2. August 187998

82. Nietzsche an Overbeck, 12. August 187999

83. Overbeck an Nietzsche, 17. August 1879100

84. Overbeck an Nietzsche, 27. August $1879 \quad 101$

85. Nietzsche an Overbeck, 27. August $1879 \quad 102$

86. Overbeck an Nietzsche, 2. September $1879 \quad 103$

87. Overbeck an Nietzsche, 9. September $1879 \quad 104$

88. Nietzsche an Overbeck, 22. September $1879 \quad 105$

89. Nietzsche an Overbeck, 29. September 1879106

90. Overbeck an Nietzsche, 29. September 1879107

91. Overbeck an Nietzsche, 13. Oktober $1879 \quad 108$

92. Nietzsche an Overbeck, 22. Oktober 1879110

93. Nietzsche an Overbeck, 24. Oktober $1879 \quad 112$

94. Nietzsche an Overbeck, 28. Oktober 1879112

95. Overbeck an Nietzsche, 1. November $1879 \quad 113$

96. Nietzsche an Ida Overbeck, 5. November 1879114

97. Ida Overbeck an Nietzsche, 11. November $1879 \quad 115$

98. Nietzsche an Overbeck, 14. November 1879116

99. Overbeck an Nietzsche, 23. November $1879 \quad 117$

100. Nietzsche an Overbeck, 11. Dezember 1879119

101. Nietzsche an Overbeck, 12. Dezember 1879120

102. Nietzsche an Overbeck, 28. Dezember 1879120

103. Overbeck an Nietzsche, 15. Januar 1880121

104. Overbeck an Nietzsche, 27. Januar 1880121

105. Nietzsche an Overbeck, Ende Januar 1880122

106. Nietzsche an Overbeck, 12. März 1880123

107. Nietzsche an Overbeck, 27. März 1880123

108. Overbeck an Nietzsche, 27. März 1880123

109. Nietzsche an Overbeck, 2. April 1880126

110. Overbeck an Nietzsche, 7. April 1880126

111. Nietzsche an Overbeck, 11. April $1880 \quad 127$

112. Overbeck an Nietzsche, 20. April $1880 \quad 128$

113. Nietzsche an Overbeck, 28. April $1880 \quad 128$

114. Nietzsche an Ida Overbeck, 24. Mai 1880129

115. Ida Overbeck an Nietzsche, 28. Mai 1880130

116. Overbeck an Nietzsche, 28. Mai 1880132

117. Nietzsche an Overbeck, 15. Juni 1880133

118. Nietzsche an Overbeck, 22. Juni 1880134

119. Nietzsche an Overbeck, 7. Juli 1880135

120. Overbeck an Nietzsche, 10. Juli 1880136

121. Nietzsche an Overbeck, 19. Juli 1880137

122. Nietzsche an Ida Overbeck, 18. August $1880 \quad 138$

123. Nietzsche an Franz und Ida Overbeck, 14. Oktober 1880140

124. Nietzsche an Franz und Ida Overbeck, 31. Oktober 1880140

125. Nietzsche an Overbeck, 17. November 1880141 
128. Nietzsche an Overbeck, 8. Januar $1881 \quad 143$

129. Nietzsche an Overbeck, 22. Februar $1881 \quad 144$

130. Nietzsche an Overbeck, 18. März $1881 \quad 145$

131. Nietzsche an Overbeck, 6. April $1881 \quad 145$

132. Nietzsche an Overbeck, 28. April 1881146

133. Nietzsche an Overbeck, 18. Mai $1881 \quad 146$

134. Nietzsche an Overbeck, 31. Mai $1881 \quad 147$

135. Nietzsche an Overbeck, 8. Juli $1881 \quad 148$

136. Nietzsche an Overbeck, 13. Juli $1881 \quad 148$

137. Nietzsche an Overbeck, 23. Juli $1881 \quad 149$

138. Nietzsche an Overbeck, 30. Juli 1881150

139. Nietzsche an Overbeck, 20./21. August $1881 \quad 150$

140. Nietzsche an Overbeck, 5. September 1881153

141. Nietzsche an Ida Overbeck, 5. September 1881154

142. Nietzsche an Overbeck, 6. September $1881 \quad 155$

143. Nietzsche an Overbeck, 18. September $1881 \quad 155$

144. Nietzsche an Overbeck, 20. September $1881 \quad 156$

145. Nietzsche an Overbeck, 14. Oktober $1881 \quad 156$

146. Nietzsche an Overbeck, 21. Oktober $1881 \quad 157$

147. Nietzsche an Overbeck, 28. Oktober $1881 \quad 158$

148. Nietzsche an Overbeck, 14. November $1881 \quad 158$

149. Nietzsche an Overbeck, 6. Dezember 1881160

150. Nietzsche an Overbeck, 12. Dezember $1881 \quad 161$

151. Nietzsche an Overbeck, 28. Dezember $1881 \quad 161$

152. Ida Overbeck an Nietzsche, 30. Dezember 1881162

153. Nietzsche an Ida Overbeck, 19. Januar 1882163

154. Nietzsche an Overbeck, 29. Januar $1882 \quad 165$

155. Overbeck an Nietzsche, 31. Januar 1882167

156. Nietzsche an Overbeck, 14. Februar 1882169

157. Nietzsche an Overbeck, Anfang März 1882170

158. Nietzsche an Overbeck, 17. März $1882 \quad 171$

159. Nietzsche an Overbeck, 17. März 1882172

160. Nietzsche an Overbeck, 8. April $1882 \quad 172$

161. Overbeck an Nietzsche, 20. April 1882173

162. Nietzsche an Overbeck, 15. Mai $1882 \quad 175$

163. Nietzsche an Overbeck, 23. Mai 1882176

164. Nietzsche an Ida Overbeck, 28. Mai 1882176

165. Nietzsche an Overbeck, etwa 5. Juni 1882177

166. Nietzsche an Overbeck, 24. Juni 1882178

167. Nietzsche an Overbeck, 18. Juli 1882179

168. Nietzsche an Overbeck, 22. August 1882180 
169. Nietzsche an Overbeck, 9. September 1882181

170. Nietzsche an Overbeck, 10. November 1882183

171. Nietzsche an Franz und Ida Overbeck, 23. November 1882 185

172. Nietzsche an Overbeck, 20. Dezember 1882186

173. Overbeck an Nietzsche, 23. Dezember 1882187

174. Nietzsche an Overbeck, 25. Dezember 1882189

175. Overbeck an Nietzsche, 27. oder 28. Dezember 1882191

176. Nietzsche an Overbeck, 31. Dezember 1882192

177. Overbeck an Nietzsche, 15. Januar 1883193

178. Nietzsche an Overbeck, 20. Januar $1883 \quad 195$

179. Overbeck an Nietzsche, 28. Januar $1883 \quad 197$

180. Nietzsche an Overbeck, 1. Februar 1883199

181. Overbeck an Nietzsche, 4. Februar 1883200

182. Nietzsche an Overbeck, 10. Februar 1883201

183. Nietzsche an Overbeck, 11. Februar 1883202

184. Nietzsche an Overbeck, 14. Februar 1883203

185. Nietzsche an Overbeck, 22. Februar 1883203

186. Nietzsche an Overbeck, 6. März 1883205

187. Nietzsche an Overbeck, 9. März 1883206

188. Nietzsche an Overbeck, 22. März 1883207

189. Overbeck an Nietzsche, 25. März 1883208

190. Nietzsche an Overbeck, Anfang April 1883212

191. Overbeck an Nietzsche, 15. April 1883213

192. Nietzsche an Overbeck, 17. April 1883215

193. Nietzsche an Overbeck, 29. April 1883217

194. Nietzsche an Overbeck, 20. Mai 1883217

195. Nietzsche an Overbeck, 15. Juni 1883219

196. Nietzsche an Overbeck, 9. Juli 1883220

197. Nietzsche an Ida Overbeck, Mitte Juli 1883221

198. Nietzsche an Ida Overbeck, 29. Juli 1883223

199. Nietzsche an Ida Overbeck, kurz vor dem 14. August 1883 226

200. Nietzsche an Overbeck, 14. August $1883 \quad 228$

201. Nietzsche an Overbeck, 18. August 1883230

202. Nietzsche an Overbeck, 26. August $1883 \quad 231$

203. Nietzsche an Overbeck, 13. Oktober 1883233

204. Nietzsche an Overbeck, 22. Oktober 1883234

205. Nietzsche an Overbeck, 27. Oktober 1883234

206. Nietzsche an Overbeck, 9. November $1883 \quad 235$

207. Overbeck an Nietzsche, 13. November $1883 \quad 237$

208. Nietzsche an Overbeck, 6. Dezember 1883239

209. Nietzsche an Overbeck, 24. Dezember 1883241 
210. Nietzsche an Overbeck, 25. Januar 1884243

211. Nietzsche an Overbeck, 6. Februar 1884244

212. Nietzsche an Overbeck, 12. Februar 1884245

213. Nietzsche an Overbeck, 8. März 1884247

214. Nietzsche an Overbeck, 28. März $1884 \quad 248$

215. Nietzsche an Overbeck, 2. April $1884 \quad 249$

216. Overbeck an Nietzsche, 3. April 1884250

217. Nietzsche an Overbeck, 7. April $1884 \quad 251$

218. Nietzsche an Overbeck, 10. April $1884 \quad 254$

219. Nietzsche an Overbeck, 30. April $1884 \quad 254$

220. Nietzsche an Overbeck, 21. Mai $1884 \quad 256$

221. Nietzsche an Overbeck, erste Juniwoche $1884 \quad 258$

222. Nietzsche an Overbeck, 12. Juli $1884 \quad 259$

223. Nietzsche an Overbeck, 18. Juli 1884261

224. Nietzsche an Overbeck, 23. Juli 1884262

225. Nietzsche an Overbeck, Anfang August 1884263

226. Nietzsche an Overbeck, 18. August 1884264

227. Nietzsche an Overbeck, 14. September 1884266

228. Nietzsche an Overbeck, 4. Oktober 1884269

229. Overbeck an Nietzsche, 7. Oktober 1884270

230. Nietzsche an Overbeck, 8. Oktober $1884 \quad 271$

231. Overbeck an Nietzsche, 13. Oktober $1884 \quad 271$

232. Nietzsche an Overbeck, 14. Oktober 1884273

233. Nietzsche an Overbeck, gegen Ende Oktober $1884 \quad 273$

234. Nietzsche an Overbeck, 6. November 1884275

235. Nietzsche an Overbeck, 13./14. November $1884 \quad 276$

236. Overbeck an Nietzsche, 21. Dezember $1884 \quad 277$

237. Nietzsche an Overbeck, 22. Dezember 1884280

238. Nietzsche an Overbeck, Anfang Januar 1885281

239. Overbeck an Nietzsche, 15. Februar 1885283

240. Nietzsche an Overbeck, 20. Februar 1885285

241. Overbeck an Nietzsche, 28. März 1885287

242. Nietzsche an Overbeck, 31. März 1885289

243. Nietzsche an Overbeck, 8. April 1885291

244. Nietzsche an Overbeck, 7. Mai 1885292

245. Nietzsche an Overbeck, 2. Juli 1885294

246. Nietzsche an Overbeck, 4. Juli 1885297

247. Nietzsche an Overbeck, 13. Juli 1885297

248. Overbeck an Nietzsche, 26. Juli 1885299

249. Nietzsche an Overbeck, 6. Oktober 1885301

250. Nietzsche an Overbeck, 7. Oktober 1885303

251. Overbeck an Nietzsche, 9. Oktober 1885304

252. Overbeck an Nietzsche, 9. Oktober 1885306

253. Nietzsche an Overbeck, 17. Oktober 1885307

254. Nietzsche an Overbeck, 12. November 1885309 
255. Overbeck an Nietzsche, 29. November 1885309

256. Nietzsche an Overbeck, Anfang Dezember 1885311

257. Overbeck an Nietzsche, 26. Dezember 1885314

258. Nietzsche an Overbeck, 9. Januar 1886317

259. Overbeck an Nietzsche, 8. März $1886 \quad 319$

260. Nietzsche an Overbeck, 25. März 1886321

261. Overbeck an Nietzsche, 29. März $1886 \quad 323$

262. Nietzsche an Overbeck, 10. April $1886 \quad 324$

263. Nietzsche an Overbeck, 25. April 1886325

264. Overbeck an Nietzsche, 29. April 1886326

265. Nietzsche an Overbeck, 1. Mai 1886328

266. Overbeck an Nietzsche, 3. Mai $1886 \quad 329$

267. Nietzsche an Overbeck, 20. Juni 1886329

268. Nietzsche an Overbeck, 28. Juni 1886331

269. Nietzsche an Overbeck, 14. Juli 1886332

270. Nietzsche an Overbeck, 5. August 1886334

271. Overbeck an Nietzsche, 22. August 1886336

272. Nietzsche an Overbeck, 12. Oktober $1886 \quad 337$

273. Nietzsche an Overbeck, 27. Oktober $1886 \quad 339$

274. Overbeck an Nietzsche, 29. Oktober $1886 \quad 340$

275. Nietzsche an Overbeck, 14. November 1886342

276. Overbeck an Nietzsche, 12. Dezember $1886 \quad 344$

277. Nietzsche an Overbeck, 25. Dezember 1886346

278. Overbeck an Nietzsche, 2. Januar $1887 \quad 347$

279. Nietzsche an Overbeck, 4. Januar $1887 \quad 349$

280. Nietzsche an Overbeck, 9. Januar 1887350

281. Nietzsche an Overbeck, 12. Februar $1887 \quad 351$

282. Nietzsche an Overbeck, 23. Februar 1887353

283. Nietzsche an Overbeck, 24. Februar 1887355

284. Overbeck an Nietzsche, 21. März 1887356

285. Nietzsche an Overbeck, 24. März 1887358

286. Nietzsche an Overbeck, 14. April 1887361

287. Nietzsche an Overbeck, 29. April 1887363

288. Nietzsche an Overbeck, 4. Mai 1887364

289. Nietzsche an Overbeck, 13. Mai 1887365

290. Nietzsche an Overbeck, 17. Juni $1887 \quad 368$

291. Nietzsche an Overbeck, 30. Juni $1887 \quad 369$

292. Overbeck an Nietzsche, 4. Juli 1887371

293. Nietzsche an Overbeck, 6. Juli 1887373

294. Nietzsche an Overbeck, 17. Juli 1887374

295. Nietzsche an Overbeck, 30. August 1887375

296. Overbeck an Nietzsche, 9. September $1887 \quad 378$

297. Nietzsche an Overbeck, 17. September 1887380

298. Nietzsche an Overbeck, 24. September 1887382 
299. Overbeck an Nietzsche, 27. September 1887

300. Nietzsche an Overbeck, 17. Oktober 1887383

301. Overbeck an Nietzsche, 24. Oktober 1887384

302. Nietzsche an Overbeck, 12. November 1887386

303. Overbeck an Nietzsche, 20. November 1887388

304. Nietzsche an Overbeck, 23. November $1887 \quad 390$

305. Nietzsche an Overbeck, 28. Dezember 1887391

306. Overbeck an Nietzsche, 2. Januar 1888391

307. Nietzsche an Overbeck, 4. Januar 1888393

308. Nietzsche an Overbeck, 3. Februar 1888394

309. Nietzsche an Overbeck, 22. Februar 1888396

310. Overbeck an Nietzsche, 1. März 1888397

311. Nietzsche an Overbeck, 3. März 1888397

312. Nietzsche an Overbeck, 22. März 1888399

313. Nietzsche an Overbeck, 10. April $1888 \quad 400$

314. Nietzsche an Overbeck, 18. April 1888402

315. Nietzsche an Overbeck, 3. Mai 1888402

316. Nietzsche an Overbeck, 27. Mai 1888403

317. Nietzsche an Overbeck, 4. Juli 1888404

318. Nietzsche an Overbeck, 11. Juli 1888406

319. Nietzsche an Overbeck, 20. Juli 1888407

320. Nietzsche an Overbeck, 26. Juli 1888409

321. Overbeck an Nietzsche, 12. September $1888 \quad 410$

322. Nietzsche an Overbeck, 14. September 1888413

323. Nietzsche an Overbeck, 9. Oktober $1888 \quad 417$

324. Overbeck an Nietzsche, 14. Oktober 1888417

325. Nietzsche an Overbeck, 18. Oktober 1888419

326. Nietzsche an Overbeck, 13. November 1888421

327. Nietzsche an Overbeck, 29. November 1888423

328. Nietzsche an Overbeck, 17. Dezember $1888 \quad 424$

329. Overbeck an Nietzsche, 21. Dezember $1888 \quad 425$

330. Nietzsche an Overbeck, 22. Dezember 1888426

331. Nietzsche an Overbeck, Weihnachten 1888428

332. Nietzsche an Overbeck, 26. Dezember 1888430

333. Nietzsche an Overbeck, 29. Dezember 1888431

334. Nietzsche an Franz und Ida Overbeck, 4. Januar 1889 